CREAT. MATH. INFORM.

Volume 31 (2022), No. 1,

Pages 71 - 79
Online version at https: //semnul .com/creative-mathematics/

Print Edition: ISSN 1584 - 286X; Online Edition: ISSN 1843 - 441X

DOI: https://doi.org/10.37193/CMI.2022.01.07

\title{
Some inequalities on the Mostar index
}

\author{
ÖZGE ÇOLAKOĞLU HAVARE
}

\begin{abstract}
Topological indices are the numerical descriptors of a molecular structure obtained via molecular graph $G$. They are used to predict physicochemical and bioactive properties of the molecules and molecular compounds. In this paper, the Mostar index is studied. It is presented the relationships between the Mostar index and other topological indices. Inequalities about some parameters and also Nordhaus-Gaddum-type results for the Mostar index are also presented.
\end{abstract}

\section{INTRODUCTION}

It has been seen that it is important to discover new drugs and vaccines to slow, stop epidemics, and also to treat many diseases in recent days. For this, it is necessary to obtain information about the physicochemical and bioactivity properties of new molecules and molecular compounds as soon as possible. Obtaining information about the properties of molecules or molecular compounds is a costly and time-consuming process. This has led to applications involving drug-like, pharmacokinetic, 3D-QSAR, molecular docking, the prediction of electronics, etc. Chemical graph theory is also focused on these applications. Topological indices, which are the subject of the chemical graph theory, are the numerical values of the molecular structures obtained via molecular graph. The vertices and edges of a molecular graph represent the atoms and the bonds between atoms, respectively. These indices are used to predict the properties of molecules such as the structure-property relationship, the structure-activity relationship, and the structural design in chemistry, nanotechnology, and pharmacology [6] .

The first applied topological index is the Wiener index and was introduced in order to calculate the boiling points of paraffin [29]. With the increasing importance of applications, many topological indices have been introduced. These indices can be classified with respect to the structural characteristics of the graph such as the degree of vertices, the distances between vertices, the matching, and the spectrum, and so on.

T. Doslic et al. introduced the Mostar index, which is a bond-additive topological index, in 2019. They gave explicit formulas for benzenoid graph, Cartesian product, extremal and unicyclic graphs [15]. A. Tepeh gave results for the Mostar index of the bicyclic graph [26]. F. Hayat and B. Zhou gave bounds on cacti with the Mostar index [22]. S. Akhter et al. computed the Mostar index of some graph operations [1]. N. Dehgardiy and M. Azariz obtained the lower bounds with the Mostar index of trees and the Mostar index of some graph operations [8]. K. Deng and S. Li presented the extremal values for the Mostar index of trees with given degree sequence [9]. K. Deng and S. Li studied the Mostar index for Chemical trees [10]. Ö. Colakoglu Havare gave the value of the Mostar index and edge Mostar index for some cycle-related graphs [6]. M. Ghorbani et al. studied on Mostar index of graphs and trees with given parameters [19]. A. Ali and T. Doslic gave known bounds and extremal results concerning the Mostar index [3].

Received: 02.09.2021. In revised form: 08.11.2021. Accepted: 15.11.2021

2010 Mathematics Subject Classification. 05C07, 05C05.

Key words and phrases. Topological index, Mostar index, Inequality, Nordhaus-Gaddum-type result.

Corresponding author: Özge Çolakoğlu Havare, ozgeeclkgl@gmail.com 
This paper focuses on relations between the Mostar index and other topological indices. It is given inequalities with other graph-theoretic parameters, like diameter, clique number for the Mostar index, and also Nordhaus-Gaddum type results for the Mostar index is given.

\section{PRELIMINARIES}

Let $G$ be a simple connected graph with a vertex set $V$ and edge set $E$. The cardinality of the vertex set of a graph is denoted by $n$ and the cardinality of its edge set is denoted by $m$. An edge of $G$ connects the vertices $u$ and $v$ and it is written by $e=u v$. The degree of a vertex $u$ is defined by $d_{u}$. The distance between vertices $u$ and $v$ is defined by $d(u, v)$. The maximum distance between all pairs of vertices of $G$ is defined the diameter of $G$, $\operatorname{diam}(G)$ [5].

First Zagrab index of a graph $G$ [21], degree-based topological index, is defined as

$$
M_{1}(G)=\sum_{u v \in E}\left(d_{u}+d_{v}\right)
$$

The Albertson of graph $G$, is known as irregularity index [2] and third Zagreb index [17] and misbalance deg index [28], is defined as

$$
\operatorname{irr}(G)=\sum_{u v \in E}\left|d_{u}-d_{v}\right| .
$$

The Wiener index of a graph $G$ [29], distance-based index, is defined as

$$
W(G)=\frac{1}{2} \sum_{v, u \subseteq V} d(u, v)=\frac{1}{2} \sum_{v \in V} d(v \mid G)
$$

where $d(v \mid G)$, is distance of a vertex $v$ of $G$, is defined as $\sum_{x \in V} d(v, x \mid G)$.

The Mostar index is defined by

$$
M o(G)=\sum_{u v \in E}\left|n_{u}-n_{v}\right|
$$

where $n_{u}$ is the number of vertices of $G$ lying closer to vertex $u$ than to vertex $v$ of the edge $u v$ [15]. $N(u)$ is the set of vertices of $G$ lying closer to vertex $u$ than vertex $v$ of the edge $u v$. Namely,

$$
N(u)=\{x \in V: d(x, u)<d(x, v)\} .
$$

The Padmakar-Ivan index of a graph $G$ is defined as [23]

$$
P I(G)=\sum_{u v \in E}\left(n_{u}+n_{v}\right) .
$$

The Szeged index of a graph $G$, first studied with the symbol $W^{*}$, is defined as (see [20]-[14])

$$
S z(G)=\sum_{u v \in E} n_{u} n_{v}
$$

A graph $G$ is said to be distance-balanced if $n_{u}=n_{v}$ for $u v \in E$. For standard terminology and notations, we follow Buckley and Harary [5].

Lemma 2.1. [18] Let $G$ be a connected graph. If $\operatorname{diam}(G)=2$ then $M o(G)=\operatorname{irr}(G)$.

Theorem 2.1. [30] Let $G$ and $\bar{G}$ be a connected graph with $n \geq 5$ and its complement, respectively. Then

$$
3\left(\begin{array}{l}
n \\
2
\end{array}\right) \leq W(G)+W(\bar{G}) \leq \frac{n^{3}+3 n^{2}+2 n-6}{6} .
$$


Lemma 2.2. [12] If $G$ is a conncected graph then

$$
\sum_{u v \in E} n_{u} n_{v}=\frac{1}{4}\left[n^{2} m-\sum_{v \in V} d(v \mid G)^{2} d_{v}+\sum_{v \in V} \sum_{u \in N(v)} d(u \mid G) d(v \mid G)\right]
$$

for $u v \in E$.

Lemma 2.3. [16] If $G$ be a conncected graph then $n_{u}-n_{v}=d(u \mid G)-d(v \mid G)$ for uv $\in E$.

Lemma 2.4. [7] Let $G$ be a connected graph with $t(G)$ triangles. Then $\sum_{u v \in E}\left|N_{u} \cap N_{v}\right|=3 t(G)$

Lemma 2.5. [27] If $a_{i} \in \mathbb{R}$ for $1 \leq i \leq n, R=\operatorname{maxa}_{i}$ and $r=$ mina $_{i}$, then

$$
n \sum_{i=1}^{n} a_{i}^{2}-\left(\sum_{i=1}^{n} a_{i}\right)^{2} \geq \frac{n}{2}(R-r)^{2}
$$

Lemma 2.6. [4] Let $a_{i}$ and $b_{i}$ are nonnegative real numbers, then

$$
\left|n \sum_{i=1}^{n} a_{i} b_{i}-\sum_{i=1}^{n} a_{i} \sum_{i=1}^{n} b_{i}\right| \leq \alpha(n)(A-a)(B-b)
$$

where $a, b, A$ and $B$ are real constants such that $a \leq a_{i} \leq A$ and $b \leq b_{i} \leq B$ for $1 \leq i \leq n$. Further, $\alpha(n)=n\left\lceil\frac{n}{2}\right\rceil\left(1-\frac{1}{n}\left\lceil\frac{n}{2}\right\rceil\right)$ with $\lceil x\rceil$ being the greatest integer function than or equal to $x$.

Lemma 2.7. [11] Let $a_{i}, b_{i}>0$ for $1 \leq i \leq n$, satisfying the condition $r a_{i} \leq b_{i} \leq R a_{i}$ for $1 \leq i \leq n$. Then

$$
r R \sum_{i=1}^{n} a_{i}^{2}+\sum_{i=1}^{n} b_{i}^{2} \leq(r+R) \sum_{i=1}^{n} a_{i} b_{i}
$$

equality holds iff $r a_{i}=b_{i}$ or $R a_{i}=b_{i}$ for $1 \leq i \leq n$.

Lemma 2.8. [25] Let $a_{i}, b_{i}>0$ for $1 \leq i \leq n$, such that $0<a \leq a_{i} \leq A$ and $0<b \leq b_{i} \leq B$ for $1 \leq i \leq n$ then

$$
\left(\sum_{i=1}^{n} a_{i}^{2}\right)\left(\sum_{i=1}^{n} b_{i}^{2}\right) \leq \frac{1}{4}\left(\sqrt{\frac{A B}{a b}}+\sqrt{\frac{a b}{A B}}\right)^{2}\left(\sum_{i=1}^{n} a_{i} b_{i}\right)^{2} .
$$

The equality holds iff $\rho=\frac{\frac{A}{a}}{\frac{A}{a}+\frac{B}{b}} n$ and $q=\frac{\frac{B}{b}}{\frac{A}{a}+\frac{B}{b}} n$ are integers, where $a_{i}=a$ for $i=1, \ldots, p$, $a_{j}=A$ for $j=p+1, \ldots, n$, and $b_{i}=b$ for $i=1, \ldots, p$, and $b_{j}=B$ for $j=p+1, \ldots, n$.

\section{INEQUALITIES ON THE Mostar INDEX}

In this section, various inequations between Mostar index and other topological indices are obtained. Moreover, the relations between the Mostar index and some paremater are presented.

Theorem 3.2. Let $G$ be a connected graph with $n$ vertices and $m$ edges. Then

$$
M o(G) \leq 2 m(n-1)-M_{1}(G) .
$$

with equality holds if and only if $G \cong S_{n}$. 
Proof. Let $n(e)$ be the number of vertices equidistant from ends of the edge $e=u v$. There is $n_{u}+n_{v}=n-n(e)$ such that $0 \leq n(e)$. It has $n_{u}+n_{v} \geq d_{u}+d_{v}$ (see [24]). Then $n-n(e) \geq d_{u}+d_{v}$. Then,

$$
\begin{aligned}
\sum_{u v \in E}\left|n_{u}-n_{v}\right| & =\sum_{u v \in E}\left|n_{u}-\left(n-n(e)-n_{u}\right)\right|=\sum_{u v \in E}\left|2 n_{u}-(n-n(e))\right| \\
& \leq \sum_{u v \in E}\left|2 n_{u}-\left(d_{u}+d_{v}\right)\right| \\
& \leq \sum_{u v \in E}\left|2(n-1)-\left(d_{u}+d_{v}\right)\right| \quad \text {, since } n_{u} \leq n-1 \\
& \leq \sum_{u v \in E} 2(n-1)-\sum_{u v \in E}\left(d_{u}+d_{v}\right), \text { since } d_{u} \leq n-1 \text { for every } u \in V .
\end{aligned}
$$

The proof is completed from the definitions of the Mostar index and the first Zagreb index. If $G$ is a star graph, then $d_{u}+d_{v}=n$ for every $u v \in E$. Hence, $M o\left(S_{n}\right)=(n-1)(n-2)=$ $\sum_{u v \in E} 2(n-1)-\sum_{u v \in E} n$.

Theorem 3.3. Let $G$ be a connected graph with $n$ vertices and $m$ edges. Then

$$
\frac{P I(G)^{2}}{m}-4 S z(G) \leq(n-2) M o(G) .
$$

The equality holds if $G$ is a star graph.

Proof. From $\left(n_{u}+n_{v}\right)^{2}-\left(n_{u}-n_{v}\right)^{2}=4 n_{u} n_{v}$ and Cauchy-Schwarz inequality, it is obtained

$$
\frac{\left(\sum_{u v \in E}\left(n_{u}+n_{v}\right)\right)^{2}}{m}-\sum_{u v \in E} 4 n_{u} n_{v} \leq \sum_{u v \in E}\left|n_{u}-n_{v}\right|^{2} .
$$

Since $\left|n_{u}-n_{v}\right| \leq n-2$, this proof is completed by using the definitions of $P I, S z$, and $M o$ indices. If $G$ is a star graph, then $\left|n_{u}-n_{v}\right|=n-2$ and from Eq. (3.2), $\frac{((n-1) n)^{2}}{n-1}-4(n-1)^{2}=$ $\sum_{u v \in E}(n-2)^{2}$.

Theorem 3.4. Let $G$ be a connected graph on $n$ vertices and $m$ edges. If graph $G$ is not distancebalanced, then

$$
P I(G)^{2}<4 m S z(G)+\frac{n^{2}-2 n+1}{4(n-2)} M o(G)^{2} .
$$

Proof. If it is chosen $a_{i}=\left|n_{u}-n_{v}\right|$ and $b_{i}=1$ such that $0<1 \leq a_{i} \leq n-2$ and $0<1 \leq$ $b_{i} \leq 1$ in Lemma 2.8, then

$$
m \sum_{u v \in E}\left|n_{u}-n_{v}\right|^{2} \leq \frac{1}{4}\left(\sqrt{n-2}+\frac{1}{\sqrt{n-2}}\right)^{2}\left(\sum_{u v \in E}\left|n_{u}-n_{v}\right|\right)^{2} .
$$

From (3.2) and (3.3), the proof is completed. If $\rho=\frac{n-2}{n-1} n$ and $q=\frac{n}{n-1}$ are integers, then this equality holds. When $n=2, \rho$ and $q$ are integers.

Theorem 3.5. Let $G$ be a connected graph on $n$ vertices and $m$ edges. If $G$ is not a distancebalanced graph, then

$$
M o(G) \geq \sqrt{2 m(n-2)} .
$$


Otherwise,

$$
m(n-2) M o(G)-M o(G)^{2} \geq \frac{m}{2}(n-2)^{2}
$$

Proof. Let $G$ be not the distance-balanced graph. If it is chosen $a_{i}=\left|n_{u}-n_{v}\right|$ such that $r=1 \leq\left|n_{u}-n_{v}\right| \leq n-2=R$ in Lemma 2.5, then the following inequality can be written

$$
m \sum_{u v \in E}\left|n_{u}-n_{v}\right|^{2}-\left(\sum_{u v \in E}\left|n_{u}-n_{v}\right|\right)^{2} \geq \frac{m}{2}(n-3)^{2} .
$$

Eq. (3.4) is obtained from (3.3) and (3.5).

Assume that $G$ be a connected graph on $n$ vertices and $m$ edges. If it is chosen $a_{i}=$ $\left|n_{u}-n_{v}\right|$ such that $0 \leq\left|n_{u}-n_{v}\right| \leq n-2$ in Lemma 2.5, it is obtained

$$
m \sum_{u v \in E}(n-2)\left|n_{u}-n_{v}\right|-\left(\sum_{u v \in E}\left|n_{u}-n_{v}\right|\right)^{2} \geq \frac{m}{2}(n-2)^{2} .
$$

From the definition of the $M o(G)$ index, the proof is completed.

Theorem 3.6. Let $G$ be a connected bipartite graph with $n$ vertices and $m$ edges. Then

$$
\frac{M o(G)^{2}}{m} \leq m n^{2}-4 S z(G) \leq(n-2) M o(G)
$$

with left equality if and ony if $G=S_{n}$ and with right equality if and only if $G=S_{n}$.

Proof. If $G$ be a connected bipartite graph, then $n_{u}+n_{v}=n$. It can be written $2 n_{u}=$ $n+n_{u}-n_{v}$ and $2 n_{v}=n+n_{v}-n_{u}$. Then,

$$
\begin{aligned}
\sum_{u v \in E} 4 n_{u} n_{v} & =\sum_{u v \in E}\left(2 n_{u} n_{u}-n_{u}^{2}-n_{v}^{2}+n^{2}\right) \\
\sum_{u v \in E}\left(n_{u}-n_{v}\right)^{2} & =\sum_{u v \in E} n^{2}-4 \sum_{u v \in E} n_{u} n_{v}
\end{aligned}
$$

From Eq. (3.6) and Chauchy-Schwarz inequality, the lower bound is obtained. From Eq. (3.6) and $\left|n_{u}-n_{v}\right| \leq n-2$, the upper bound is obtained.

Theorem 3.7. Let $G$ be a connceted graph with $n$ vertices and $m$ edges. Then

$$
\left|\frac{\left(P I(G)^{2}+n M o(G)^{2}\right)}{(n+1)}-P I(G) M o(G)\right|<\gamma(m)(n-2)^{2}
$$

where $\gamma(m)=m\left\lceil\frac{m}{2}\right\rceil\left(1-\frac{1}{m}\left\lceil\frac{m}{2}\right\rceil\right)$.

Proof. If it is choosen $a_{i}=\left|n_{u}-n_{v}\right|$ and $b_{i}=\left(n_{u}+n_{v}\right)$ such that $0 \leq a_{i} \leq n-2$ and $2 \leq b_{i} \leq n$ in Lemma 2.6, then the following inequality can be written

$$
\left|m \sum_{u v \in E}\right| n_{u}^{2}-n_{v}^{2}\left|-\sum_{u v \in E}\right| n_{u}-n_{v}\left|\sum_{u v \in E}\left(n_{u}+n_{v}\right)\right| \leq \gamma(m)(n-2)(n-2) .
$$

If $a_{i}=\left|n_{u}-n_{v}\right|$ and $b_{i}=\left(n_{u}+n_{v}\right)$ such that $\left|n_{u}-n_{v}\right| \leq\left(n_{u}+n_{v}\right) \leq n\left|n_{u}-n_{v}\right|$ in Lemma 2.7 are chosen, then it is obtained

$$
n \sum_{u v \in E}\left|n_{u}-n_{v}\right|^{2}+\sum_{u v \in E}\left|n_{u}+n_{v}\right|^{2} \leq(n+1) \sum_{u v \in E}\left|n_{u}^{2}-n_{v}^{2}\right| .
$$

From combining (3.7) with (3.8), it is obtained that $\left|\frac{m}{n+1}\left(n \sum_{u v \in E}\left|n_{u}-n_{v}\right|^{2}+\sum_{u v \in E}\left|n_{u}+n_{v}\right|^{2}\right)-\sum_{u v \in E}\right| n_{u}-n_{v}\left|\sum_{u v \in E}\right| n_{u}+n_{v}|| \leq \gamma(m)(n-2)^{2}$. 
When $\left|n_{u}-n_{v}\right|=\left(n_{u}+n_{v}\right)$, there is equality. By using the definitions of $M o(G)$ and $P I(G)$ indices and Cauchy-Schwarz inequality, this proof is completed.

Theorem 3.8. If $G$ is connected graph with maximum vertex degree $\Delta$, then

$$
M o(G)<2 \Delta W(G) .
$$

Proof. From Lemma 2.3, it can be written

$$
\begin{aligned}
M o(G) & =\sum_{u v \in E}|d(u \mid G)-d(v \mid G)|=\frac{1}{2} \sum_{v \in V} \sum_{u \in N(v)}|d(u \mid G)-d(v \mid G)| \\
& \leq \frac{1}{2} \sum_{v \in V} \sum_{u \in N(v)}|d(u \mid G)|+\frac{1}{2} \sum_{v \in V} \sum_{u \in N(v)}|d(v \mid G)| \\
& =\frac{1}{2} \sum_{v \in V} d(v \mid G) d_{v}+\frac{1}{2} \sum_{v \in V} d(v \mid G) d_{v} \\
& <\Delta \sum_{v \in V} d(v \mid G)=2 \Delta W(G)
\end{aligned}
$$

which completes the proof.

Theorem 3.9. If $G$ is a connected graph with $n$ vertices, $m$ edges, maximum vertex degree $\Delta$, and diameter $\operatorname{diam}(G)$, then

$$
M o(G)^{2}<4 m \Delta(n-1) \operatorname{diam}(G) W(G)+4 m S z(G)-n^{2} m^{2} .
$$

Proof. By using Lemma 2.3, it can be written

$$
\begin{aligned}
\sum_{u v \in E}\left|n_{u}-n_{v}\right|^{2} & =\sum_{u v \in E}|d(u \mid G)-d(v \mid G)|^{2}=\frac{1}{2} \sum_{v \in V} \sum_{u \in N(v)}|d(u \mid G)-d(v \mid G)|^{2} \\
& =\sum_{v \in V} d(v \mid G)^{2} d_{v}-\sum_{v \in V} \sum_{u \in N(v)} d(u \mid G) d(v \mid G) \\
& =\sum_{v \in V} d(v \mid G)^{2} d_{v}+4 \sum_{u v \in E} n_{u} n_{v}-n^{2} m+\sum_{v \in V} d(v \mid G)^{2} d_{v} \text {, since Lemma } 2.2
\end{aligned}
$$

By using Cauchy-Schwarz inequality and $d(v \mid G)<(n-1) \operatorname{diam}(G)$, it is obtained

$$
\frac{\left(\sum_{u v \in E}\left|n_{u}-n_{v}\right|\right)^{2}}{m}<2 \Delta(n-1) \operatorname{diam}(G) \sum_{v \in V} d(v \mid G)+4 \sum_{u v \in E} n_{u} n_{v}-n^{2} m
$$

By using definitions, the proof is completed.

Corollary 3.1. Let $T$ be a tree graph with $n$ vertices. Then

$$
M o(T)^{2}<(n-1)(2 n-4) W(T) .
$$

Proof. If $G$ is a tree graph, then

$$
\sum_{u \in N(v)} d(u \mid G)=d(v \mid G) d_{v}+n d_{v}-2(n-1) .
$$


From equations (3.9) and (3.10),

$$
\begin{aligned}
\sum_{u v \in E}\left|n_{u}-n_{v}\right|^{2} & =\sum_{v \in V} d(v \mid G)\left[d(v \mid G) d_{v}-d(v \mid G) d_{v}-n d_{v}+2(n-1)\right] \\
& =\sum_{v \in V} d(v \mid G)\left[-n d_{v}+2(n-1)\right]
\end{aligned}
$$

If $G$ is a tree graph then minimum degree is 1 . Then,

$$
\sum_{u v \in E}\left|n_{u}-n_{v}\right|^{2}<2(n-2) W(T) .
$$

From Cauchy-Schwarz inequality, this proof is completed.

Theorem 3.10. Let $G$ be a connected graph with $n$ vertices, $m$ edges and diameter $\operatorname{diam}(G)$. If $G$ is not a distance-balanced, then

$$
M o(G) \geq\left\lfloor\frac{\operatorname{diam}(G)^{2}}{2}\right\rfloor+m-\operatorname{diam}(G)
$$

with equality holds if and only if $G \cong P_{n}$.

Proof. If $G$ is not a distance-balanced, then $\left|n_{u}-n_{v}\right| \geq 1$ for $u v \in E$. Since $G$ has diameter $\operatorname{diam}(G)$, the path $P_{\operatorname{diam}(G)+1}$ is a subgraph $G$. Then

$$
\begin{aligned}
M o(G) & \geq \sum_{u v \in E\left(P_{\operatorname{diam}(G)+1}\right)}\left|n_{u}-n_{v}\right|+\sum_{u v \in E \backslash E\left(P_{\operatorname{diam}(G)+1}\right)}\left|n_{u}-n_{v}\right| \\
& \geq \operatorname{Mo}\left(P_{\operatorname{diam}(G)+1}\right)+(m-\operatorname{diam}(G)) 1 .
\end{aligned}
$$

If $m=\operatorname{diam}(G)$ then $M o(G) \geq M o\left(P_{\operatorname{diam}(G)+1}\right)$ and also the equality is hold when $n=$ $\operatorname{diam}(G)+1$ and thus $G$ is isomorphic to the $P_{n}$.

Theorem 3.11. Let $G$ be a connected graph with $n$ vertices, $m$ edges and clique $\omega(G)$. If $G$ is not a distance-balanced graph, then

$$
M o(G) \geq m-\frac{\omega(G)(\omega(G)-1)}{2}
$$

with equality if and only if $G$ is isomorphic to the $K_{n}-e$.

Proof. Let $G$ be not distance-balanced. Then, $\left|n_{u}-n_{v}\right| \geq 1$ and also $M o(G) \geq 1$. Denote the clique of graph $G$ by graph $K_{\omega}$. Hence, graph $G$ contains graph $K_{\omega}$. Then,

$$
M o(G) \geq \sum_{u v \in E\left(K_{\omega}\right)}\left|n_{u}-n_{v}\right|+\sum_{u v \in E \backslash E\left(K_{\omega}\right)}\left|n_{u}-n_{v}\right|=\left(m-\frac{\omega(G)(\omega(G)-1)}{2}\right) 1 .
$$

Since $M o(G) \geq 1, m \geq \frac{\omega(G)(\omega(G)-1)}{2}+1$. When $m=\frac{\omega(G)(\omega(G)-1)}{2}+1$, the equality is holds. Hence, $G$ is isomorphic to the $K_{n}-e$.

Theorem 3.12. If $G$ is connected graph with $n$ vertices, $m$ vertices and $t(G)$ triangles, then

$$
M o(G) \leq m n-2 m+3 t(G)
$$

with equality if and only if $G$ is isomorphic $S_{n}$. 
Proof. Since $n_{u}+n_{v} \leq n-t(G)$, it can be written

$$
\begin{aligned}
M o(G) & \leq \sum_{u v \in E}\left|n-n_{v}-t(G)-n_{v}\right| \\
& \leq \sum_{u v \in E}\left|n-2 n_{v}\right|+\sum_{u v \in E}|t(G)| \\
& \leq \sum_{u v \in E}|n-2 n+2|+\sum_{u v \in E}|t(G)| \quad \text { since } n_{v} \leq n-1 \\
& \leq m(n-2)+3 t(G) \quad \text { since Lemma 2.4. }
\end{aligned}
$$

If $G$ is a bipartite graph then, $n_{u}+n_{v}=n$ and $t(G)=0$ and hence $M o(G) \leq m(n-2)=$ $\sum_{u v \in E}|n-1-(1)|$. Then, if $G$ is a star graph with $n$ vertices then the equality is hold.

Theorem 3.13. Let $G$ be a distance-balanced and connected graph with $n \geq 5$. If $\bar{G}$ is connected complement of $G$, then

$\left\lfloor\frac{\operatorname{diam}(G)^{2}}{2}\right\rfloor+\left(\begin{array}{c}n \\ 2\end{array}\right)+\frac{1}{2}(\operatorname{diam}(G)-3) \operatorname{diam}(G) \leq M o(G)+M o(\bar{G}) \leq \frac{n^{4}+3 n^{3}+2 n^{2}-6 n}{3}$ with left equality if and ony if $G=P_{n}$.

Proof. From Theorem 3.8, it can be written

$$
\begin{aligned}
M o(G)+M o(\bar{G}) & \leq 2 \Delta W(G)+2 \bar{\Delta} W(\bar{G}) \\
& \leq 2 n(W(G)+W(\bar{G})) \\
& \leq 2 n \frac{n^{3}+3 n^{2}+2 n-6}{6} \quad \text { from Theorem 2.1 }
\end{aligned}
$$

which completes the lower bound of the proof.

Let $\overline{P_{n}}$ be the complement of the path graph. The vertices degree of the path graph are 1 or 2 . So, vertices degrees of $\overline{P_{n}}$ are $n-2$ or $n-3$ and $\operatorname{diam}\left(\overline{P_{n}}\right)=2$. From Lemma 2.1, the following equation is obtained

$$
M o\left(\overline{P_{n}}\right)=2(n-2)+(n-2)(n-3) .
$$

From inequation (3.11) and Eq. (3.12),

$$
\begin{aligned}
M o(\bar{G}) & \geq \sum_{u v \in E\left(\bar{P}_{\operatorname{diam}(G)+1}\right)}\left|n_{u}-n_{v}\right|+\sum_{u v \in E \backslash E\left(\bar{P}_{\operatorname{diam}(G)+1}\right)}\left|n_{u}-n_{v}\right| \\
& =\operatorname{Mo}\left(\bar{P}_{\operatorname{diam}(G)+1}\right)+\bar{m}-\frac{1}{2}(\operatorname{diam}(G)-1) \operatorname{diam}(G) \\
& =(\operatorname{diam}(G)-1) \operatorname{diam}(G)+\left(\begin{array}{c}
n \\
2
\end{array}\right)-m-\frac{1}{2}(\operatorname{diam}(G)-1) \operatorname{diam}(G)
\end{aligned}
$$

From Theorem 3.10 and Eq.(3.13) the proof is completed.

\section{CONCLUSION}

This paper gives new inequalities for the Mostar index. Relationships between the Mostar index and the Padmakar-Ivan, the Szeged, the Wiener, and the first Zagreb indices are found. Boundaries are obtained between the diameter, clique, and triangle parameters of a $G$ graph and the Mostar index of a $G$ graph. In addition, Nordhaus-Gaddum-type results are given for the Mostar index of a distance-balanced $G$ graph. The results of this paper will be a light for the applications of the Mostar index. 


\section{REFERENCES}

[1] Akhter, S.; Iqbal, Z.; Aslam, A.; Gao, W. Computation of Mostar index for some graph operations. Int. J. Quantum Chem. 121 (2021), no. 15, e26674.

[2] Albertson, M. O. The irregularity of a graph. Ars Comb. 46 (1997), 219-225.

[3] Ali, A.; Došlić, T. Mostar index: Results and perspectives. Appl Math Comp. 404 (2021), 1262457.

[4] Biernacki, M.; Pidek, H.; Ryll-Nardzewsk, C. Sur une iné galité entre des intégrals dénies. Maria Curie SkÁC odowska Univ. A4 (1950), 1-4.

[5] Buckley, F.; Harary, F. Distance in Graphs. Addison-Wesley Publishing Company, Advanced Book Program, Redwood City, CA, 1990.

[6] Çolakoğlu Havare, Ö. Mostar Index (Mo) and Edge Mo Index for Some Cycle Related Graphs. Romanian J. Math. Comp. Sci. 10 (2020), no. 1, 53-66.

[7] Das, K. Ch.; Gutman, I. Estimating the Szeged index. Appl Math. Lett. 22 (2009), 1680-1684.

[8] Dehgardiy, N.; Azariz, M. More On Mostar Index. Appl. Math. E-Notes 20 (2020), 316-322.

[9] Deng, K.; Li, S. On the extremal values for the Mostar index of trees with given degree sequence. Appl Math Comput. 390 (2021), 125598.

[10] Deng, K.; Li, S. Chemical Trees with Extremal Mostar Index. MATCH Commun. Math. Comput. Chem. 85 (2021), 161-180.

[11] Diaz, B.; Metcalf, T. Stronger forms of a class of inequalities of G. Bull. Am. Math. Soc. 69 (1963), $415-418$.

[12] Dobrynin, A.; Gutman, I. On a graph invariant related to the sum of all distances in a graph. Publ. Inst. Math.(Publications de L'institut mathematique) (Beograd), 56 (1994), no. 70, 18-22.

[13] Dobrynin, A.; Gutman, I. Solving a problem connected with distances in graphs. Graph Theory Notes, New York 28 (1995), 21-23.

[14] Dobrynin, A. A.; Gutman, I.; DSmStSr, G. A Wiener-type graph invariant for some bipartite graphs. Appl. Math. Lett. 8 (1995), no. 5,57-62.

[15] Doslic, T.; Martinjak, I.; Skrekovski, R.; Spuzevic, S. T.; Zubac, I. Mostar Index. J. Math. Chem. (2018), 1-19, Doi: 10.1007/s10910-018-0928-z.

[16] Entringer, R. C.; Jackson, D. E.; Snyder, D. A. Distance in graphs. Czechoslovak Math. J. 26 (1976), $283-296$.

[17] Fath-Tabar, G. H. Old and New Zagreb Indices of Graphs. MATCH Commun. Math. Comput. Chem. 65 (2011), 79-84.

[18] Gao, F.; Xu, K.; Doslic, T. On the Difference of Mostar Index and Irregularity of Graphs. Bull. Malays. Math. Sci. Soc. 44 (2021), 905-926, https:/ / doi.org/10.1007/s40840-020-00991-y.

[19] Ghorbani, M.; Rahmani, S.; Eslampoor, MJ. Some New Results on Mostar Index of Graphs. Iranian J. Math. Chem. 11 (2020), no. 1, 33-42.

[20] Gutman, I. A formula for the Wiener number of trees and its extension to graphs containing cycles. Graph Theory Notes, New York 27 (1994), 9-15.

[21] Gutman, I.; Trinajstic, N. Graph theory and molecular orbitals, Total electron energy of alternant hydrocarbons. Chem. Phys. Lett. 17 (1972), 535-538.

[22] Hayat, F.; Zhou, B. On Cacti with Large Mostar Index. Filomat 33 (2019), no. 15, 4865-4873, https://doi.org/10.2298/FIL1915865H.

[23] Khadikar, P. V. On novel structural descriptor PI. Nat. Sci. Lett. 23 (2000), 113-118.

[24] Mogharrab, M.; Maimani, H. R.; Ashrafi, A. R. A note on the vertex PI index of graphs. J. Adv. Math. Studies. 2 (2009), no. 2, 53-56.

[25] Polya, G.; Szegö, G. Problems and Theorems in Analysis I: Series, Integral Calculus, Theory of Functions. Springer, Verlag, New York, 1972.

[26] Tepeh, A. Extremal bicyclic graphs with respect to Mostar index. Appl. Math. Comput. 355 (2019), 319-324.

[27] von Szökefalvi Nagy, J. Über algebraische Gleichungen mit lauter reellen Wurzeln. Jahresbericht der Deutschen Mathematiker-Vereinigung 27 (1918), 37-43.

[28] Vukičević, D.; Gašperov, M. Bond Additive Modeling 1. Adriatic Indices. Croat. Chem. Acta 83 (2010), no. 3, 243-260.

[29] Wiener H. Structural Determination of Parafin Boiling Points. J. American Chem. Soc. 69 (1947), 17-20.

[30] Zhang, L.; Wu, B. The Nordhaus-Gaddum-type inequalities for some chemical indices. MATCH Commun. Math. Comput. Chem. 54 (2005), 183-194.

MERSIN UNIVERSITY

MATHEMATICS DEPARTMENT

33343, MERSIN, TURKEY

Email address: ozgeeclkgl@gmail.com 